\title{
Association Between Adherence to the Mediterranean Diet and Asthma in Peruvian Children
}

\author{
Jessica L. Rice ${ }^{1} \cdot K_{\text {Karina M. Romero }}{ }^{2}$ Rocio M. Galvez Davila ${ }^{2}$ Carla Tarazona Meza $^{2} \cdot$ \\ Andrew Bilderback ${ }^{3}$. D'Ann L. Williams ${ }^{4}$ - Patrick N. Breysse ${ }^{4} \cdot$ Sonali Bose $^{3}$. \\ William Checkley ${ }^{3,4,5} \cdot$ Nadia N. Hansel ${ }^{3,4}$ - GASP Study Investigators
}

Received: 29 June 2015/Accepted: 19 August 2015/Published online: 3 September 2015

(C) The Author(s) 2015. This article is published with open access at Springerlink.com

\begin{abstract}
Purpose Adherence to a Mediterranean diet pattern may be associated with lower asthma prevalence in children. We sought to corroborate these findings in Peruvian children.

Methods This case-control study included children of ages 9-19 years living in Lima, Peru. A food frequency questionnaire (FFQ) was completed and diet pattern was analyzed using a modified Mediterranean diet score (MDS). Primary analysis investigated the relationship between MDS and asthma status. Maternal education, age, sex, and body mass index category were included in multivariate model. Secondary outcomes included asthma control, forced expiratory volume in $1 \mathrm{~s}$ (FEV1), allergic rhinitis, and atopic status.

Results 287 participants with asthma and 96 controls without asthma completed a FFQ. Mean age was
\end{abstract}

Nadia N. Hansel

nhansel1@jhmi.edu

1 Division of Pediatric Pulmonology, School of Medicine, Johns Hopkins University, Baltimore, MD, USA

2 Biomedical Research Unit, A.B. PRISMA, Lima, Peru

3 Division of Pulmonary and Critical Care, School of Medicine, Johns Hopkins University, Baltimore, MD, USA

4 Department of Environmental Health Sciences, Bloomberg School of Public Health, Johns Hopkins University, Baltimore, MD, USA

5 Program in Global Disease Epidemiology and Control, Department of International Health, Bloomberg School of Public Health, Johns Hopkins University, Baltimore, MD, USA
13.5 years. According to the asthma control test (ACT), $86 \%$ of those with asthma were controlled (score $>19$ ). MDS scores ranged 6-18 (median 15). In adjusted analysis, being above the median MDS scores was associated with decreased odds of asthma [OR $=0.55,95 \%$ CI $(0.33$, $0.92), p=0.02]$. Among children whose mothers completed secondary education, being above the median MDS significantly decreased the odds of asthma $[\mathrm{OR}=0.31$, $95 \%$ CI $(0.14,0.71), p<0.01]$, whereas among those whose mothers did not complete secondary education there was no protective effect $[\mathrm{OR}=0.86,95 \% \mathrm{CI}(0.43,1.7)$, $p=0.66]$. There was no association between MDS scores and asthma control, FEV1, allergic rhinitis, or atopic status. Conclusion Adherence to the Mediterranean diet was inversely associated with having asthma among children in Lima, Peru. This effect was strongest among children with better educated mothers.

Keywords Peru - Allergic rhinitis - Atopic - Control · Food frequency questionnaire $\cdot$ Diet pattern

$\begin{array}{ll}\text { Abbreviations } \\ \text { MDS } & \text { Mediterranean diet score } \\ \text { FFQ } & \text { Food frequency questionnaire } \\ \text { BMI } & \text { Body mass index } \\ \text { ACT } & \text { Asthma control test } \\ \text { FEV }_{1} & \text { Forced expiratory volume in one second } \\ \text { FVC } & \text { Forced vital capacity } \\ \text { ISAAC } & \text { International Study of Asthma and Allergies in } \\ & \text { Childhood } \\ \text { ATS } & \text { American Thoracic Society } \\ \text { OR } & \text { Odds ratio } \\ \text { SES } & \text { Socioeconomic status } \\ \text { NAEPP } & \text { National Asthma Education and Prevention } \\ & \text { Program }\end{array}$




\section{Introduction}

Asthma and related allergic diseases are significant public health problems worldwide [1,2]. Changes in the western diet, which is deficient in antioxidants, are postulated to be one of the reasons for the asthma epidemic [3].

The traditional Mediterranean diet provides food sources rich in antioxidants such as vegetables, legumes, fruits and nuts, cereals, fish, a high monounsaturated-to-saturated fat ratio mostly from olive oil, low-fat dairy products, and a low intake of meat and poultry [4]. This diet pattern has been shown to be protective of asthma [5-7] and allergic disease [7-9] in multiple cross-sectional observational studies. Most of these studies have been completed in Mediterranean countries where the diet is similar to the traditional pattern. In this current study, our goal was to corroborate these findings in a non-Mediterranean population of Latino children in Peru.

In Peru, asthma morbidity is a major health problem with rates above global average for reported asthma and current wheeze [10]. We compared the Peruvian diet to a Mediterranean diet pattern and investigated the relationship between the Mediterranean diet and asthma prevalence and control. We hypothesized that high adherence to a Mediterranean diet would be associated with a lower likelihood of asthma and better symptom control among children in Lima, Peru.

\section{Methods}

\section{Study Setting}

Participants included children enrolled in a case-control study investigating environmental and genetic associations with asthma. We recruited children 9-19 years of age who lived in the Pampas zone of San Juan de Miraflores in Lima, Peru. Asthma cases and controls were selected from a registry collected during a local census conducted by A.B. PRISMA in 2010. Eligible participants were selected at random, and the parent or legal guardian was consented and children signed an assent form. The study was approved by the Internal Review Boards at Johns Hopkins School of Medicine in Baltimore, USA and at A.B. PRISMA in Lima, Peru.

\section{Study Design}

Participants with asthma were those who were ever diagnosed with asthma by a physician. Those with current asthma also had asthma-like symptoms (wheezing, dyspnea at rest or nocturnal dyspnea) and/or used bronchodilators, and/or inhaled corticosteroids in the past 12 months. Participants with a chronic respiratory condition other than asthma as well as those who were pregnant were excluded. Inclusion criteria for control participants included those who had never been diagnosed by a physician with asthma, had no symptoms consistent with asthma or use of asthma medications in past year, and had normal spirometry ( $\mathrm{FEV}_{1}$ $>80 \%$ predicted and $\mathrm{FEV}_{1} / \mathrm{FVC}$ ratio $>85 \%$ ).

\section{Data Collection}

Questionnaires were interviewer administered to both the participant and caregiver to document asthma status and assess respiratory symptoms using standard, Spanish-translated and validated questions from the International Study of Asthma and Allergies in Childhood (ISAAC) questionnaire [11]. A health survey also collected information on 1) demographics, including age, gender, caregiver education; 2) comorbid illnesses associated with asthma (i.e., allergic rhinitis); and 3) medication use. Asthma control was assessed using the validated Spanish-translated ACT [12]. Asthma controller use was defined as having a physician prescribed inhaled corticosteroid. Current allergic rhinitis was defined as having allergic rhinitis symptoms (sneezing or a runny or blocked nose) in the last 12 months.

Height and weight were measured, and body mass index (BMI) was calculated in $\mathrm{kg} / \mathrm{m}^{2}$. Our analysis used cut-off points for BMI in childhood that are based on international data and linked to the widely accepted adult cut-off points of a body mass index of 25 and $30 \mathrm{~kg} / \mathrm{m}$ [13, 14]. A flowbased portable spirometer (SpiroPro, Jaeger/ERT, Hoechberg, Germany) was used to measure Forced Vital Capacity (FVC), FEV1, and FEV1/FVC. We obtained at least three acceptable and reproducible spirometric maneuvers in accordance to the American Thoracic Society (ATS) and European Respiratory Society guidelines [15]. We used height-adjusted criteria derived by Hankinson and colleagues to obtain predicted values for FEV1 and FVC [16]. We tested all patients for reversibility with 4 puffs from a salbutamol inhaler ( $90 \mathrm{mcg} / \mathrm{puff}$ ), and reversibility was defined as increase in FEV1 of $\geq 12 \%$ or an increase $\geq 10 \%$ of predicted FEV1, consistent with National Asthma Education and Prevention Program (NAEPP-3) guidelines [17].

An ImmunoCAP 250 (Thermo Fisher Scientific Inc.) in vitro assay to three allergen panels (animal epidermal and proteins mix, house dust mix, and a mold and yeast mix) was used to determine atopic status. A positive result (IgE level $>0.1 \mathrm{kUa} / \mathrm{L}$ ) for any of the three allergen mixes was considered atopic. We had complete data to define atopic status in $76 \%(n=291)$ of our participants.

Field staff administered a FFQ, which was a Peruvian diet-specific 170-item questionnaire that assessed usual 
dietary intake over the previous 2 weeks, including 20 food groups and beverages. Of the 342 children with asthma, all were invited to complete the FFQ and 287 (84\%) participated. Of the 290 without asthma in the parent study, 96 (33\%) were randomly selected to complete a FFQ. The food item list was developed from 24-h recalls collected from 60 respondents who were similar in age, socioeconomic status (SES), and neighborhood to our study population. Responses include never and 1-2, 3-5, 6-9, 10-13, and 14 or more times during the last 2 weeks. A modified MDS score developed by Castro-Rodriguez et al. [18] was used, which was based on the scoring system of Psaltopoulou et al. [19]. Fruit, fish, vegetables, legumes, cereals, pasta, rice, and potatoes were considered pro-Mediterranean foods and rated according to the frequency of intake ( 0 points $=$ never or occasionally; 1 point $\geq 1-2$ times/ week; 2 points $\geq 3$ times/week) [18]. Since the study involved children, we presumed milk to be positive and not detrimental $[8,20]$. Meat, sugary drinks, and fast food were considered anti-Mediterranean foods and were rated inversely ( 0 points $\geq 3$ times/week; 1 point $\geq 1-2$ times/ week; 2 points $=$ never or occasionally) [18]. Candies, industrial pastry, precooked pizzas, and fried food, along with hamburgers were classified generically as fast food [18]. Scores could range from 0 to 22 with a higher score meaning that the diet was more adherent to the Mediterranean diet.

\section{Statistical Analysis}

Statistical analyses of differences between children with and without asthma were performed using the Chi-squared test for categorical variables and $t$ test for continuous variables. Logistic regression was used to calculate odds ratios (OR) and $95 \%$ confidence intervals. We dichotomized MDS above and below the median (15). The primary analyses investigated the relationship between MDS and asthma status (asthma vs. control) using multivariate logistic regression after adjusting for potential confounders, including age, sex, BMI category, and maternal education as an indicator of SES. Interactions between MDS and maternal education, BMI category, age and sex were tested in multivariate models. A significant interaction was found between MDS and maternal education (interaction $p=0.03$ ) and multivariate models were subsequently stratified by maternal education (completed secondary education vs. did not complete secondary education).

Secondary analyses evaluated the relationship between MDS and asthma control (controlled $=$ ACT score $>19$ vs. uncontrolled = ACT $\leq 19$ ), atopic status, and the presence of allergic rhinitis using logistic regression and for $\mathrm{FEV}_{1}$ percent predicted using linear regression for the whole group and for asthmatics and controls separately. Multivariate models of asthma control did not include adjustment for asthma controller medication use as few participants utilized controller medications (2\%). There was no significant interaction between MDS and maternal education for our secondary outcomes (asthma control, atopy, allergic rhinitis, and $\mathrm{FEV}_{1}$ ) and so final models were not stratified. All analyses were performed using Stata 13.1 statistical software (Stata Corp, College Station, TX).

\section{Results}

\section{Participant Characteristics}

A total of 383 participants completed a FFQ, 287 asthma cases and 96 controls. Mean age was 13.5 (SD 2.6) years with no difference between those with and without asthma. Median MDS score was 15. Children with asthma were less likely to be female, more likely to have mothers who completed secondary school education, and had higher rates of atopy compared to control subjects (Table 1). Of those with asthma, $86 \%$ had controlled asthma (ACT score $>19)$ and only $6(2 \%)$ children reported having a prescribed inhaled corticosteroid.

Comparing those who completed the FFQ with those who did not and were therefore not included in this analysis, there was a significant difference in age among controls (13.3 vs. 14.0 years, $p=0.04$ ), but no significant difference in sex, BMI, or maternal education. Among those with asthma, there was no difference in asthma severity or ACT score.

\section{Association of Mediterranean Diet Score with Asthma Status}

In bivariate analysis, being above the median MDS was associated with a decreased odds of asthma diagnosis [OR $0.56,95 \% \mathrm{CI}(0.36,0.90), p=0.02]$. In adjusted analysis, this relationship remained statistically significant, [OR $0.55,95 \%$ CI $(0.33,0.92), p=0.02)]$. A significant interaction was found between MDS and maternal education (interaction $p=0.03$ ), and adjusted models were further stratified by maternal education (completed vs. did not complete secondary education). Among children whose mothers had completed secondary education, higher MDS scores were associated with a decreased odds of asthma [OR 0.31, $95 \%$ CI (0.14, 0.71), $p<0.01$ ]; however, among children whose mother had not completed secondary education, there was no statistically significant association between MDS and odds of asthma (Table 2; Fig. 1). 
Table 1 Participant characteristics

\begin{tabular}{|c|c|c|c|}
\hline & $\begin{array}{l}\text { Asthma } \\
N=287\end{array}$ & $\begin{array}{l}\text { Control } \\
N=96\end{array}$ & $p$ value \\
\hline Female, $n(\%)$ & $112(39)$ & $56(58)$ & $<0.01$ \\
\hline Age, mean (SD) & $13.5(2.6)$ & $13.4(2.4)$ & 0.60 \\
\hline BMI category, $n(\%)(n=383)$ & & & 0.20 \\
\hline Underweight & 0 & 0 & \\
\hline Normal weight & $176(61)$ & $68(71)$ & \\
\hline Overweight & $80(28)$ & $22(23)$ & \\
\hline Obese & $31(11)$ & $6(6)$ & \\
\hline Maternal education: secondary school or higher $(n=364)$ & $164(59)$ & $33(38)$ & $<0.01$ \\
\hline MDS (above median), $n(\%)$ & $118(41)$ & $53(55)$ & 0.02 \\
\hline Atopic $(n=291)$ & $186(77 \%)$ & $23(47 \%)$ & $<0.01$ \\
\hline Current allergic rhinitis, $n(\%)(n=365)$ & $158(57)$ & $20(22)$ & $<0.01$ \\
\hline $\mathrm{FEV}_{1}$ percent predicted, mean (SD) & $106(14)$ & $110(14)$ & $<0.01$ \\
\hline $\mathrm{FEV}_{1} / \mathrm{FVC}$ percent predicted, mean (SD) & $87(7)$ & $91(4)$ & $<0.01$ \\
\hline $\mathrm{FEV}_{1}$ reversibility, $\mathrm{n}(\%)$ & $45(16)$ & 0 & $<0.01$ \\
\hline ACT score, mean (SD) & $22.7(3.2)$ & & \\
\hline$\%$ with controlled asthma $(\mathrm{ACT}>19)$ & 86 & & \\
\hline
\end{tabular}

Table 2 Association between MDS and outcomes

\section{Crude OR (95\% CI), $p$ value}

Adjusted $^{\text {a }}$ OR (95 \% CI), $p$ value

\section{Asthma \\ Overall group \\ Allergic rhinitis \\ Atopy \\ Overall group \\ Asthma group \\ Control group \\ Asthma control \\ Overall group \\ Asthma group}

High maternal education

Low maternal education

$\mathrm{FEV}_{1}$ percent predicted

$$
\begin{aligned}
& 0.56(0.36,0.90), p=0.02 \\
& 0.33(0.15,0.74), p<0.01 \\
& 0.78(0.40,1.49), p=0.45 \\
& 0.77(0.51,1.16), p=0.21
\end{aligned}
$$$$
0.85(0.51,1.42), p=0.53
$$$$
0.82(0.45,1.49), p=0.52
$$$$
1.3(0.41,3.92), p=0.67
$$$$
\beta 0.84(-2.1,3.8), p=0.58
$$$$
\beta 0.18(-3.2,3.6), p=0.92
$$$$
0.78(0.40,1.52), p=0.46
$$

$$
\begin{aligned}
& 0.55(0.33,0.92), p=0.02 \\
& 0.31(0.14,0.71), p<0.01 \\
& 0.86(0.43,1.7), p=0.66 \\
& 0.75(0.49,1.15), p=0.18 \\
& 0.83(0.49,1.41), p=0.49 \\
& 0.82(0.44,1.53), p=0.53 \\
& 1.5(0.45,5.17), p=0.50 \\
& 0.70(0.35,1.38), p=0.3 \\
& \beta 1.77(-1.2,4.7), p=0.24 \\
& \beta 0.28(-3.18,3.73), p=0.87
\end{aligned}
$$

${ }^{a}$ Adjusted for BMI category, sex, age, maternal education; maternal education not included in models stratified by maternal education status

\section{Association of Mediterranean Diet Score with Asthma Control (ACT), FEV 1, Atopy, and Allergic Rhinitis}

Among those with current asthma, MDS score was not associated with asthma control status. Specifically, having an MDS above the median was not associated with greater odds of having controlled asthma (ACT $>19$ ) in bivariate [OR $0.78,95 \% \mathrm{CI}(0.40,1.52), p=0.46$ ] or multivariate [OR $0.7095 \%$ CI $(0.35,1.38), p=0.3$ ] analyses. In sensitivity analyses, after adjusting for atopic status as well as $\mathrm{FEV}_{1}$ percent predicted, there was still no significant effect of MDS on asthma control (data not shown). We did not find a significant relationship between MDS and $\mathrm{FEV}_{1}$ percent predicted, atopic status or the presence of allergic rhinitis symptoms among the group overall, or among asthma or control groups (Table 2).

\section{Discussion}

In this study, we compared a Peruvian diet to the traditional Mediterranean diet and examined the relationship between diet pattern and asthma status. We found that among children in Peru, moderate adherence to the Mediterranean diet was associated with a lower odds of having asthma. 
Fig. 1 Odds of asthma if MDS is above versus below the median among children whose mother did or did not complete secondary school. Maternal secondary education completed: OR 0.31 (95\% CI 0.14, 0.71), $p<0.01$. Maternal secondary education not completed: OR 0.86 (95\% CI 0.43, 1.7), $p=0.66$

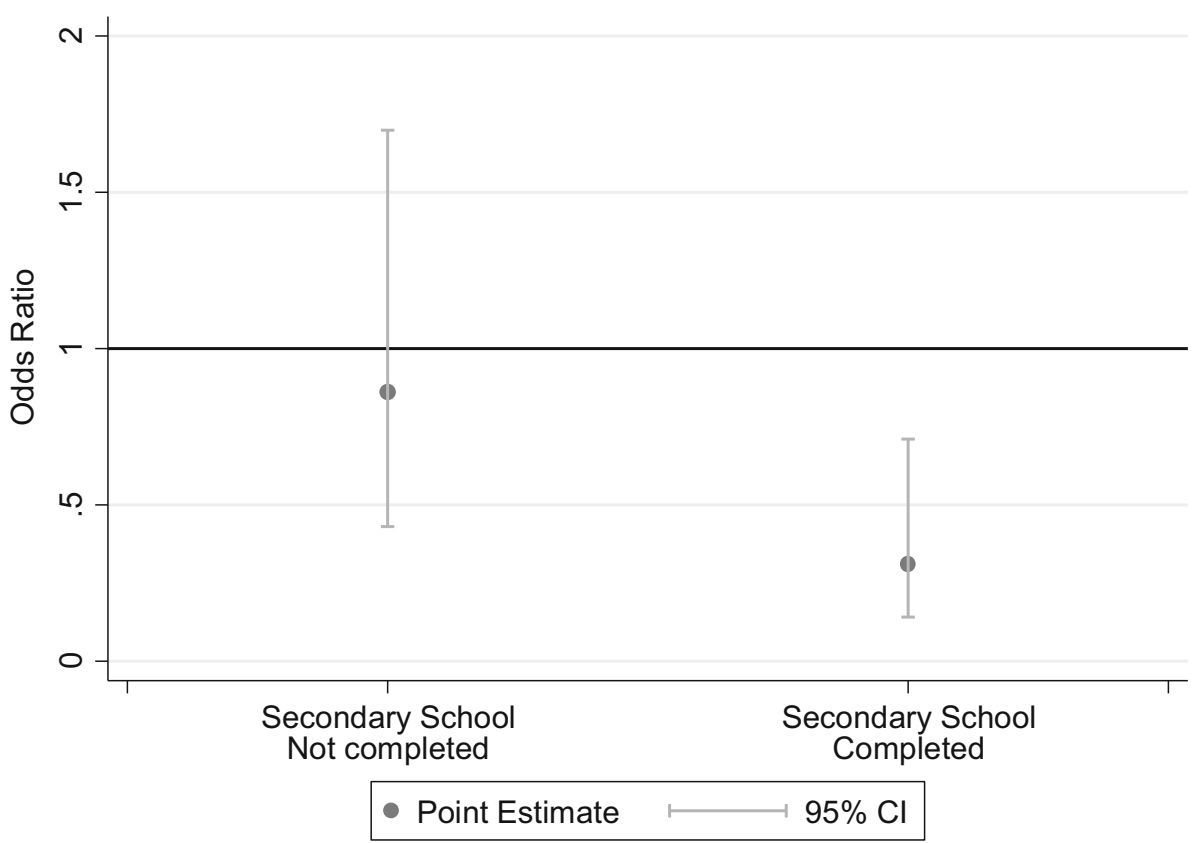

This relationship was strongest among children whose mothers have completed secondary education. While many epidemiological studies have found a similar protective effect of a Mediterranean diet on asthma in children [5-7, 21], others have not replicated this finding [22]. Our results are consistent with the recent systematic review and metaanalysis of eight studies which showed that being in the highest tertile of MDS during childhood is a protective factor for ever being diagnosed with asthma [23].

Our Peruvian children had MDS scores that ranged from 6 to 18 (scale range $0-22$ ) with a median of 15 . These values were similar to what other studies have found using the same scale and showed that the Peruvian children studied had even slightly higher adherence to a Mediterranean diet than children studied in Spain. Garcia-Marcos used the same MDS in Spanish school children and noted a median of 13 points (range 4-20) [24]. Gonzalez Barcala also studied Spanish children and had a median of 13 (range 3-21) in the 6-7 year olds and 12 (range 4-20) in the 13-14-year-old group [22].

Adherence to a Mediterranean diet was not associated with better asthma control among those with asthma in our study. A majority of the children with asthma were controlled according to the ACT $(86 \%)$ and so the study may have been limited in detecting a difference. In Portugal, Barros et al. found that in adults, high adherence to the Mediterranean diet was associated with asthma control (defined as $\mathrm{FEV}_{1} \geq 80 \%$ of predicted, exhaled nitric oxide $\leq 35 \mathrm{ppb}$, and Asthma Control Questionnaire score $<1$ ) [25]. None of the available studies in children have specifically evaluated asthma control and its relationship to the Mediterranean diet pattern; however, in the systematic review of epidemiological studies assessing Mediterranean diet and prevalence of 'current wheeze,' there was a significant negative association with the highest compared with the lowest tertile of MDS [OR $0.85,95 \%$ CI $(0.75$, 0.98), $p=0.02$ ] [23].

In our analysis, we did not find a significant relationship between MDS and atopic status or allergic rhinitis among the asthma or control groups. Similar to our results, the ISAAC ( $n=50,004)$ did not find an association between MDS and allergic sensitization [6]; however, other results suggest that MDS adherence may be related to decreased prevalence of atopy or allergic rhinitis. In Crete, Chatzi et al. noted that adherence to the Mediterranean diet was associated with a decreased odds of allergic rhinitis [8]. Similarly, De Batlle et al. found a negative relationship between being in the two higher tertiles of MDS and allergic rhinitis ever, current sneezing, and itchy-watery eyes [7].

Maternal education was used as a marker of SES, and though it is not clear why adherence to a Mediterranean diet is only protective of asthma in children with more educated mothers, there are several plausible explanations. Perhaps these parents made overall healthier diet and lifestyle choices for their families, or children of mothers with less education may have other factors common in environments of lower SES that outweigh the relevance of diet in contributing to asthma development, such as possible increased exposure to pollution and increased stress, which are recognized contributors to asthma morbidity [26-29].

There are no Mediterranean diet intervention trials in children, and studies supplementing the diet with 
antioxidants have had mixed results [30, 31]. In a 12-week open-label trial by Sexton et al., 38 adults with current asthma were randomized to a high intervention group ( $41 \mathrm{~h}$ of dietician services), low intervention group ( $2 \mathrm{~h}$ of services), or control (one dietician session and recipes) [32]. The study achieved its primary outcome of altering the eating habits of participants in the high intensity intervention toward a Mediterranean diet pattern [32]. The study was not powered to detect clinical endpoints but did note statistically non-significant improvements in asthmarelated quality of life and spirometry in the intervention groups [32]. There was no effect on asthma control [32].

Our study had several limitations. The FFQ can be associated with recall bias and as with all cross-sectional studies; we are unable to show the time relationships of the associations found, so it is not possible to reach causal conclusions. While there is no validated FFQ in our population, the current FFQ was developed from $24 \mathrm{~h}$ recalls collected in our Peruvian community. This FFQ is effective at ranking participants relative to each other in terms of the intake frequencies that were used to calculate MDS. The FFQ does not allow correction for energy intake, but there was no difference in BMI categories between those with and without asthma. Furthermore, dietary scores estimating adherence to a Mediterranean diet have been developed, but there is no validated MDS available for use in children. Our scoring system and analysis of the MDS was a modified one used in pediatric populations in other studies [6, $18,22,24]$.

\section{Conclusions}

Our results show an inverse association between adherence to a Mediterranean diet and odds of asthma among children in Lima, Peru suggesting a protective role of Mediterranean diet on the development of asthma. We did not find an association between high adherence to the Mediterranean diet and level of disease control, $\mathrm{FEV}_{1}$, allergic rhinitis, or atopic status. In conclusion, diet may be an important, modifiable risk factor for the development of asthma in children, and the present findings need to be confirmed by interventional or prospective studies prior to recommending intake of the Mediterranean diet for asthma prevention or treatment.

Acknowledgments We would like to acknowledge study investigators and staff of the Genetic susceptibility to Asthma and pollution in Peru (GASP) Study: Patrick N. Breysse, William Checkley, Rocio M. Galvez Davila, Gregory B Diette, Rafael Guerrero-Preston, Nadia N. Hansel, Caroline Johnson, John Lima, Gary Malpartida, Ed Mougey, Jesse Negherbon, Suzanne Pollard, Karina M. Romero, Carla Tarazona, Lindsay Underhill, D'Ann L. Williams. The Genetic susceptibility to Asthma and pollution in Peru (GASP) Study was supported by NIEHS R01ES018845.

\section{Compliance with Ethical Standards}

Conflict of interest The authors report no conflicts of interest. The authors alone are responsible for the content and writing of the paper.

Ethical approval All procedures performed in studies involving human participants were in accordance with the ethical standards of the institutional and/or national research committee and with the 1964 Helsinki declaration and its later amendments or comparable ethical standards.

Open Access This article is distributed under the terms of the Creative Commons Attribution 4.0 International License (http://crea tivecommons.org/licenses/by/4.0/), which permits unrestricted use, distribution, and reproduction in any medium, provided you give appropriate credit to the original author(s) and the source, provide a link to the Creative Commons license, and indicate if changes were made.

\section{References}

1. Masoli M, Fabian D, Holt S, Beasley R (2004) Global Burden of Asthma Report. Global Initiative for Asthma. University of Southampton and Medical Research Institute of New Zealand

2. Centers for Disease Control and Prevention (2013) Asthma Facts-CDC's National Asthma Control Program Grantees. U.S. Department of Health and Human Services, Centers for Disease Control and Prevention, Atlanta, GA

3. Devereux G, Seaton A (2005) Diet as a risk factor for atopy and asthma. J Allergy Clin Immunol 115(6):1109-1117. doi:10.1016/ j.jaci.2004.12.1139. quiz 1118

4. Trichopoulou A, Costacou T, Bamia C et al (2003) Adherence to a Mediterranean diet and survival in a Greek population. N Engl J Med 348(26):2599-2608. doi:10.1056/NEJMoa025039

5. Arvaniti F, Priftis KN, Papadimitriou A et al (2011) Adherence to the Mediterranean type of diet is associated with lower prevalence of asthma symptoms, among 10-12 years old children: the PANACEA study. Pediatr Allergy Immunol 22(3):283-289. doi:10.1111/j.1399-3038.2010.01113.x

6. Nagel G, Weinmayr G, Kleiner A et al (2010) Effect of diet on asthma and allergic sensitisation in the International Study on Allergies and Asthma in Childhood (ISAAC) Phase Two. Thorax 65(6):516-522. doi:10.1136/thx.2009.128256

7. de Batlle J, Garcia-Aymerich J, Barraza-Villarreal A et al (2008) Mediterranean diet is associated with reduced asthma and rhinitis in Mexican children. Allergy 63(10):1310-1316. doi:10.1111/j. 1398-9995.2008.01722.x

8. Chatzi L, Apostolaki G, Bibakis I et al (2007) Protective effect of fruits, vegetables and the Mediterranean diet on asthma and allergies among children in Crete. Thorax 62(8):677-683. doi:10. 1136/thx.2006.069419

9. Chatzi L, Torrent M, Romieu I et al (2008) Mediterranean diet in pregnancy is protective for wheeze and atopy in childhood. Thorax 63(6):507-513. doi:10.1136/thx.2007.081745

10. The Global Asthma Report (2011) The International Union Against Tuberculosis and Lung Disease, Paris, France

11. Asher MI, Keil U, Anderson HR et al (1995) International Study of Asthma and Allergies in Childhood (ISAAC): rationale and methods. Eur Respir J 8(3):483-491

12. Nathan RA, Sorkness CA, Kosinski M et al (2004) Development of the asthma control test: a survey for assessing asthma control. J Allergy Clin Immunol 113(1):59-65. doi:10.1016/j.jaci.2003. 09.008 
13. Cole TJ, Bellizzi MC, Flegal KM et al (2000) Establishing a standard definition for child overweight and obesity worldwide: international survey. BMJ 320(7244):1240-1243

14. WHO Expert Committee (1995) Physical status: the use and interpretation of anthropometry. Report of a WHO Expert Committee. World Health Organ Tech Rep Ser 854:1-452

15. Miller MR, Hankinson J, Brusasco V et al (2005) Standardisation of spirometry. Eur Respir J 26(2):319-338. doi:10.1183/ 09031936.05.00034805

16. Quanjer PH, Stanojevic S, Cole TJ et al (2012) Multi-ethnic reference values for spirometry for the 3-95-yr age range: the global lung function 2012 equations. Eur Respir J 40(6):1324-1343. doi: $10.1183 / 09031936.00080312$

17. Expert Panel Report 3 (EPR-3): (2007) Guidelines for the Diagnosis and Management of Asthma-Summary Report 2007. J Allergy Clin Immunol 120(5 Suppl):S94-S138. doi:10.1016/j. jaci.2007.09.043

18. Castro-Rodriguez JA, Garcia-Marcos L, Alfonseda Rojas JD et al (2008) Mediterranean diet as a protective factor for wheezing in preschool children. J Pediatr 152(6):823-828, 828.e821-822. doi:10.1016/j.jpeds.2008.01.003

19. Psaltopoulou T, Naska A, Orfanos P et al (2004) Olive oil, the Mediterranean diet, and arterial blood pressure: the Greek European Prospective Investigation into Cancer and Nutrition (EPIC) study. Am J Clin Nutr 80(4):1012-1018

20. Jennings A, Welch A, van Sluijs EM et al (2011) Diet quality is independently associated with weight status in children aged 9-10 years. J Nutr 141(3):453-459. doi:10.3945/jn.110.131441

21. Grigoropoulou D, Priftis KN, Yannakoulia M et al (2011) Urban environment adherence to the Mediterranean diet and prevalence of asthma symptoms among 10- to 12-year-old children: the Physical Activity, Nutrition, and Allergies in Children Examined in Athens study. Allergy Asthma Proc 32(5):351-358. doi:10. 2500/aap.2011.32.3463

22. Gonzalez Barcala FJ, Pertega S, Bamonde L et al (2010) Mediterranean diet and asthma in Spanish schoolchildren. Pediatr Allergy Immunol 21(7):1021-1027. doi:10.1111/j.1399-3038. 2010.01080.x
23. Garcia-Marcos L, Castro-Rodriguez JA, Weinmayr G et al (2013) Influence of Mediterranean diet on asthma in children: a systematic review and meta-analysis. Pediatr Allergy Immunol 24(4):330-338. doi:10.1111/pai.12071

24. Garcia-Marcos L, Canflanca IM, Garrido JB et al (2007) Relationship of asthma and rhinoconjunctivitis with obesity, exercise and Mediterranean diet in Spanish schoolchildren. Thorax 62(6):503-508. doi:10.1136/thx.2006.060020

25. Barros R, Moreira A, Fonseca J et al (2008) Adherence to the Mediterranean diet and fresh fruit intake are associated with improved asthma control. Allergy 63(7):917-923. doi:10.1111/j. 1398-9995.2008.01665.x

26. Yonas MA, Lange NE, Celedon JC (2012) Psychosocial stress and asthma morbidity. Curr Opin Allergy Clin Immunol 12(2):202-210. doi:10.1097/ACI.0b013e32835090c9

27. Forno E, Celedon JC (2009) Asthma and ethnic minorities: socioeconomic status and beyond. Curr Opin Allergy Clin Immunol 9(2):154-160

28. Beck AF, Huang B, Simmons JM et al (2014) Role of financial and social hardships in asthma racial disparities. Pediatrics 133(3):431-439. doi:10.1542/peds.2013-2437

29. Kopel LS, Phipatanakul W, Gaffin JM (2014) Social Disadvantage and Asthma Control in Children. Paediatr Respir Rev 15(3):256-263. doi:10.1016/j.prrv.2014.04.017

30. Thien FCK, De Luca S, Woods RK, Abramson MJ (2002) Dietary marine fatty acids (fish oil) for asthma in adults and children. Cochrane Database Syst Rev. doi:10.1002/14651858. CD001283

31. Milan SJ, Hart A, Wilkinson M (2013) Vitamin C for asthma and exercise-induced bronchoconstriction. Cochrane Database Syst Rev 10:CD010391. doi:10.1002/14651858.CD010391.pub2

32. Sexton P, Black P, Metcalf P et al (2013) Influence of Mediterranean diet on asthma symptoms, lung function, and systemic inflammation: a randomized controlled trial. J Asthma 50(1):75-81. doi:10.3109/02770903.2012.740120 